\title{
Advantages of Trichloro Iso Cyanuric Acid over Commercially Available Liquid Chlorine for Sanitation of Swimming Pools in Nagpur Region
}

\author{
Lanje war Pras hant S. Smt Radhikatai ${ }^{1,{ }^{*}}$, Parbat D. K. ${ }^{2}$, Kos ankar P. T. ${ }^{3}$ \\ ${ }^{1}$ Pandav College of En gin eering, Umrer Road, bahadura, Nagpur \\ ${ }^{2}$ Govt. Polytechnic, Sakoli \\ ${ }^{3}$ Yashwantrao Chavhan College of Engineering, Hin gana, Nagpur
}

\begin{abstract}
This research paper highlights on disinfection and sanitation studies on swimming pools based on several experiments carried out in different swimming pools including water parks in Nagpur and Suburbs. It also suggests practical and effective methods of swimming pool sanitation using TCCA in lieu of existing Liquid chlorine sources. Once implemented, the pool management could have savings potential up to Rs 4,000 per month per ONE Lakh litre capacity pool
\end{abstract}

Keywords Swimming Pool Sanitation Engineering, Disinfection

\section{The Objectives}

Nagpur the central Region of India has seen continuous rise of swimming pools and water parks. The Region experiences primarily three main seasons - summer, winter and monsoon with wide fluctuations in temperature. Almost throughout the summer, starting March; the temperature remains beyond 40 degree Celsius. The Chemistry of water in the pool is an ever-changing environment that calls for constant and careful monitoring. Pool San itation, especially, is recognized as a means of controlling communicable diseases. The pool operators are expected to provide safe and clean water for bathers. Water can become aggressive and destroy pools with corrosion, or it can become scaling and damage the pool with mineral deposits. The pool operator must learn about the use of chemical agents for sanitation and for control of $\mathrm{pH}$, total alka lin ity and calcium hardness (1). The pool operators utilise Chlorine gas and Liquid Chlorine as the main source for sanitation. During winter season, the escaped chlorine from the pool surface create environmental issues. Neighbours complain about skin and eye irritations. Being an industrial problem, without much referred work done, there had been a need to find an effective solution to this environmental problem. The objective of these experiments is to carry out "comparative studies on chlorinating agents - Liquid Chlorine and TCCA 90 ; to be more specific in winter season in swimming pools and water

* Corresponding author:

pslanjewar@yahoo.com (Lanjewar Prashant S. Smt Radhikatai)

Published online at http://journal.sapub.org/ajee

Copyright (C) 2012 Scientific \& Academic Publishing. All Rights Reserved parks in Nagpur region" and recommend necessary dosagelevels. Free Available Chlorine should be maintained in pools at 1 to $3 \mathrm{ppm}$ - preferably $2 \mathrm{ppm}$ but not less than 1 ppm

Sodium Hypochlorite solution or commercial liquid bleach containing $12.5 \%$ available chlorine ( practically close to $10 \%$ at the use point ) is preferred by pool operators due to it's advantages like being the lowest or next to gas cheapest available chlorine source. Sodium Hypochlorite does not require dissolution, neither leaves residues and can be used with chemical feed pumps. However it has it's disadvantages of being bulky and heavy to handle , un stabilized nature, rapid loss of strength, higher $\mathrm{pH}(10-13)$ thereby raising the $\mathrm{pH}$ of pool.

Chlorine is extremely susceptible to sunlight and needs to be regularly monitored. But just as we use sunscreen to protect our skin from the sun, chlorine uses a sunscreen of Cyanuric acid ( 2 ). Used this way, Cyanuric acid is also commonly called a stabilizer or conditioner. Cyanuric acid is used in outdoor pools along with the inorganic chlorine (3) for effective sanitations. Advantages and disadvantages of the most popular chlorine based Cyanuric acid product called Tri - Chloro - Iso Cyanuric acid with $90 \%$ available Chlorine ( TCCA 90 ) is given below

TCCA - 90 : Trichlor oisocyanuric Acid: Granules or tablets $90 \%$ available ch lorine

\begin{tabular}{|c|c|}
\hline Advan tages: & Disadvantages: \\
\hline Stabilized - chlorine doesn't dissipate & Cost slight ly higher \\
\hline Easy to handle & Lowers pH - pH 2.8 \\
\hline Low cost, low maintenance erosion feeders & Lowers total alkalinity \\
\hline $\begin{array}{c}\text { Highly concentrated - } 90 \% \text { available } \\
\text { chlorine }\end{array}$ & $\begin{array}{c}\text { May elevate Cyanuric } \\
\text { acid levels }\end{array}$ \\
\hline Dissolves completely & \\
\hline
\end{tabular}




\section{Reccomended Water Saturation Summary in Pools}

Properly balanced or saturated water prevents damage to the pool and equipment. Unsaturated water corrodes plaster walls, fixtures, plumbing, etc., and causes staining. Oversat urated water deposits scale or becomes cloudy. The operator needs to test and control Free Available Chlorine, $\mathrm{pH}$, total alkalinity, TDS, \& hardness to maintain balanced water.

- Free Available Chlorine should be maintained at 1 to 3 ppm - preferably 2 ppm

- Proper pH control (7.2 to 7.6) pre ferably 7.4 that ensures: (1) the proper form of carbonate alka lin ity for saturation, and (2) the proper form of HOCI for sanitizer efficacy. Soda ash is used to raise $\mathrm{pH}$. Acids are used to reduce $\mathrm{pH} \&$ total alkalinity.

- Total Alkalinity of $90-120 \mathrm{ppm}$ is preferred for $\mathrm{pH}$ buffering \& calcium carbonate saturation. Sodium bicarbonate is used to raise total alka lin ity.

- Calcium hardness of 270 to $360 \mathrm{ppm}$ is preferred for proper calcium carbonate saturation and for avoiding soft-water scale found in pools. Calcium chloride is used to raise calcium hardness.

- TDS below $1200 \mathrm{ppm}$ is recommended for pools and water parks

- Total Mic robial load should not exceed $100 \mathrm{CFU} / \mathrm{ml}$ of water

\section{Methodology}

Sanitation is the process of destroying organisms referred to as pathogens, including bacteria, fungi, viruses, etc. Chlorination also controls algae - which are not usually harmful themselves, but may harbor pathogenic organisms. As part of overall studies plan, these set of experiments are carried out in various swimming pools and water parks in and around Nagpur in winter season to study the effectiveness. During these studies pools traditionally being operated with Liquid Chlorine are evaluated. The details of the pools under study are given in Table \# 1 . There are five pools coded as AB 05, PK 06, PK 07, PC 13 and CG 14 using Liquid Chlorine, out of which PC 13 was evaluated with TCCA - 90 for comparis on purposes .

The water park pools under this study were shut down for maintenance in Rainy Season and were to be opened up to public from $2^{\text {nd }}$ October 2010 onwards. Similarly the Public swimming pool was to be offered to public fro $m 2^{\text {nd }}$ October 2010 onwards. The authors took it up as an opportunity to study efficacies of Liquid chlorine and TCCA in these pools.

Analys is ${ }^{(4)}$ : Free Available Chlorine ( FAC) has to be analysed immed iately after sampling. There are 25 samples drawn from five pools at periodic intervals for Analysis. The nearest location of a laboratory is $40-50 \mathrm{KM}$ away, talking a couple of hours to reach. The proximity of analys is centre for FAC does not make it feasible to carry samples for analysis. Hence standard method used for commercial estimation of FAC using Ortho Toludiene reagent was used for estimation of FAC. During the process $10 \mathrm{ml}$ pool water is taken in a test tube to which $10 \mathrm{ml}$ Ortho Toludiene solution is added. The contents are shaken and sample is compared in Chloroscope for getting FAC Value.

The climatic condition details are reproduced from the Climatological unit of India Meteorological Department, Nagpur centre.

Chlorinating agent is added in the evening at $7 \mathrm{PM}$ and FAC readings are taken the subsequent days at $8 \mathrm{AM}, 12$ Noon, 2 PM, 4 PM and 6 PM. Water park pools are initially evaluated with Liquid Chlorine. However the Resort pool PC 13 is tried out with TCCA. The observations for Pools are given in Table \# 2 to Table \# 6 respectively.

Table 1. Pool details

\begin{tabular}{|c|c|c|c|c|}
\hline Sr No & Pool Description & Pool Code & Pool Capacity Liters & Remarks \\
\hline 01 & Water Park Pools & AB 05 & $\mathbf{3 0 0 , 0 0 0 ~ L i t e r s ~}$ & Liquid Chlorine Operated \\
\hline 02 & & PK 06 & $\mathbf{4 0 0 , 0 0 0 ~ L i t e r s ~}$ & Liquid Chlorine Operated \\
\hline 03 & Re sort Pool & PK 07 & $\mathbf{5 0 0 , 0 0 0 ~ L i t e r s ~}$ & Liquid Chlorine Operated \\
\hline 04 & Public Swimming Pools & CG 14 13 & $\mathbf{3 5 0 , 0 0 0 ~ L i t e r s ~}$ & TCCA 90 (90.0\% Available Chlorine ) \\
\hline 05 & 600,000 Liters & Liquid Chlorine Operated \\
\hline
\end{tabular}

Table 2. Monitoring of FAC in Park Pool : AB 05 ( Liquid Chlorine Operated)

\begin{tabular}{|c|c|c|c|c|c|c|c|}
\hline Pool Capacity KL & 300 & & & & & & \\
\hline Chlorinat ing Agent & Liqui & orine & & & & & \\
\hline Run No & $\mathrm{R} \# 001$ & $\mathrm{R} \# 002$ & $\mathrm{R} \quad \# 003$ & $\mathrm{R} \# 004$ & $\mathrm{R} \# 005$ & $\mathrm{R} \# 006$ & $\mathrm{R} \# 007$ \\
\hline Run Date (Year 2010) & Sep-24 & Sep-25 & Sep-26 & Sep-27 & Sep-28 & Sep-29 & Sep-30 \\
\hline Maximum Temp Deg C & 43.0 & 43.0 & 44.3 & 43.1 & 44.1 & 45.6 & 47.1 \\
\hline Minimum T emp Deg C & 22.5 & 24.0 & 24.0 & 24.9 & 24.5 & 25.6 & 27.6 \\
\hline Relative Humidity $\%$ Evening & 65 & 100 & 63 & 60 & 67 & 55 & 57 \\
\hline Wind Velocity Km/Hr Evening & 8.0 & 14.0 & 4.0 & 4.0 & 8.0 & 6.0 & 6.0 \\
\hline Rains if any / Remarks & & Rainy & Rainy & & Cloudy & & \\
\hline Time ofLiquid Chlorine addition & 7:00 PM & 7:00 PM & 7:00 PM & 7:00 PM & 7:00 PM & 7:00 PM & 7:00 PM \\
\hline Quantity Added Kg & 70 & 70 & 70 & 70 & 35 & 35 & 35 \\
\hline Theoretical Cl2 ppm & 29.2 & 29.2 & 29.2 & 29.2 & 14.6 & 14.6 & 14.6 \\
\hline MeasuredCl2@8 AM Next day & & 3 & 2.8 & 2.8 & 2.8 & 2.8 & 2.8 \\
\hline Measured Cl2@12 Noon & & 2 & 2.2 & 2.2 & 2.4 & 2.4 & 2.6 \\
\hline Measured Cl2@2PM & & 1.4 & 1.8 & 2 & 1.8 & 1.6 & 1.8 \\
\hline MeasuredC2@4PM & & 0.6 & 1.2 & 1.4 & 1.2 & 1.2 & 1.4 \\
\hline Measured C22@6PM & & 0.2 & 0.4 & 0.6 & 0.8 & 0.8 & 1 \\
\hline
\end{tabular}


Table 3. Monitoring of FAC in Park Pool : PK 06 ( Liquid Chlorine Operated )

\begin{tabular}{|c|c|c|c|c|c|c|c|}
\hline Pool Capacity KL & \multicolumn{2}{|c|}{400} & & & & & \\
\hline Chlorinating Agent & \multicolumn{2}{|c|}{ Liquid Chlorine } & & & & & \\
\hline Run No & $\mathrm{R} \# 008$ & $\mathrm{R} \# 009$ & $\mathrm{R} \# 010$ & $\mathrm{R} \# 011$ & $\mathrm{R} \# 012$ & $\mathrm{R} \# 013$ & $\mathrm{R} \# 014$ \\
\hline Run Date (Year 2010) & Sep-24 & Sep-25 & Sep-26 & Sep-27 & Sep-28 & Sep-29 & Sep-30 \\
\hline Maximum Temp Deg C & 43.0 & 43.0 & 44.3 & 43.1 & 44.1 & 45.6 & 47.1 \\
\hline Minimum Temp Deg C & 22.5 & 24.0 & 24.0 & 24.9 & 24.5 & 25.6 & 27.6 \\
\hline Relative Humidity \% Evening & 65 & 100 & 63 & 60 & 67 & 55 & 57 \\
\hline Wind Velocity Km/Hr Evening & 8.0 & 14.0 & 4.0 & 4.0 & 8.0 & 6.0 & 6.0 \\
\hline Rains if any / Remarks & & Rainy & Rainy & & Cloudy & & \\
\hline Time of Liquid Chlorine addition & $7: 00$ PM & $7: 00$ PM & $7: 00$ PM & $7: 00$ PM & $7: 00$ PM & $7: 00$ PM & $7: 00$ PM \\
\hline Quantity Added Kg & 70 & 70 & 70 & 70 & 35 & 35 & 35 \\
\hline Theoretical C12 ppm & 21.9 & 21.9 & 21.9 & 21.9 & 10.9 & 10.9 & 10.9 \\
\hline Measured C2 @ 8 AM Next day & 2.8 & 2.8 & 2.8 & 2.6 & 2.6 & 2.8 \\
\hline Measured C2 @ 12 Noon & & 1.8 & 2 & 2.2 & 2 & 2 & 2.2 \\
\hline Measured C2 @ 2 PM & & 1 & 1.2 & 1.4 & 1.8 & 1.8 & 1.8 \\
\hline Measured C12 @ 4 PM & & 0.4 & 0.6 & 1 & 1.4 & 1.2 & 1.4 \\
\hline Measured C12 @ 6 PM & & 0.2 & 0.4 & 0.6 & 0.8 & 1 & 1.2 \\
\hline
\end{tabular}

Table 4. Monitoring of FAC in Park Pool : PK 07 ( Liquid Chlorine Operated )

\begin{tabular}{|c|c|c|c|c|c|c|c|}
\hline Pool Capacity KL & 500 & & & & & & \\
\hline Chlorinating Agent & \multicolumn{2}{|c|}{ Liquid Chlorine } & & & & & \\
\hline Run No & $\mathrm{R} \# 015$ & $\mathrm{R} \# 016$ & $\mathrm{R} \# 017$ & $\mathrm{R} \# 018$ & $\mathrm{R} \# 019$ & $\mathrm{R} \# 020$ & $\mathrm{R} \# 021$ \\
\hline Run Date (Year 2010) & Sep-24 & Sep-25 & Sep-26 & Sep-27 & Sep-28 & Sep-29 & Sep-30 \\
\hline Maximum Temp Deg C & 43.0 & 43.0 & 44.3 & 43.1 & 44.1 & 45.6 & 47.1 \\
\hline Minimum Temp Deg C & 22.5 & 24.0 & 24.0 & 24.9 & 24.5 & 25.6 & 27.6 \\
\hline Relative Humidity \% Evening & 65 & 100 & 63 & 60 & 67 & 55 & 57 \\
\hline Wind Velocity $\mathrm{Km} / \mathrm{Hr}$ Evening & 8.0 & 14.0 & 4.0 & 4.0 & 8.0 & 6.0 & 6.0 \\
\hline Rains if any / Remarks & & Rainy & Rainy & & Cloudy & & \\
\hline Time of Liquid Chlorine addition & 7:00 PM & 7:00 PM & 7:00 PM & 7:00 PM & 7:00 PM & 7:00 PM & 7:00 PM \\
\hline Quantity Added Kg & 105 & 105 & 70 & 35 & 35 & 70 & 70 \\
\hline Theoretical $\mathrm{Cl} 2 \mathrm{ppm}$ & 26.3 & 26.3 & 17.5 & 8.8 & 8.8 & 17.5 & 17.5 \\
\hline \multicolumn{2}{|c|}{ Measured C12@8 AM Next day } & 2.8 & 2.8 & 2.4 & 2.6 & 2.8 & 2.8 \\
\hline Measured C2 @12 Noon & & 2.0 & 1.8 & 1.4 & 1.8 & 2.0 & 2.2 \\
\hline Measured Cl2@2PM & & 1.2 & 1.4 & 0.6 & 0.8 & 1.4 & 1.8 \\
\hline Measured Cl2@4PM & & 0.2 & 0.6 & 0.2 & 0.4 & 1.2 & 1.4 \\
\hline Measured C12@6PM & & 0.1 & 0.4 & 0.1 & 0.2 & 0.8 & 1.0 \\
\hline
\end{tabular}

Table 5. Monitoring of FAC in Resort Pool :PC 13 ( TCCA Operated)

\begin{tabular}{|c|c|c|c|c|c|c|c|}
\hline Pool Capacity KL & 350 & & & & & & \\
\hline Chlorinating Agent & \multicolumn{4}{|c|}{ TCCA 90 ( $90 \%$ Available Chlorine $)$} & & & \\
\hline Run No & $\mathrm{R} \# 022$ & $\mathrm{R} \# 023$ & $\mathrm{R} \# 024$ & $\mathrm{R} \# 025$ & $\mathrm{R} \# 026$ & $\mathrm{R} \# 027$ & $\mathrm{R} \# 028$ \\
\hline Run Date (Year 2010) & Sep-24 & Sep-25 & Sep-26 & Sep-27 & Sep-28 & Sep-29 & Sep-30 \\
\hline Maximum Temp Deg C & 43.0 & 43.0 & 44.3 & 43.1 & 44.1 & 45.6 & 47.1 \\
\hline Minimum Temp Deg C & 22.5 & 24.0 & 24.0 & 24.9 & 24.5 & 25.6 & 27.6 \\
\hline Relative Humidity \% Evening & 65 & 100 & 63 & 60 & 67 & 55 & 57 \\
\hline Wind Velocity $\mathrm{Km} / \mathrm{Hr}$ Evening & 8.0 & 14.0 & 4.0 & 4.0 & 8.0 & 6.0 & 6.0 \\
\hline Rains if any / Remarks & & Rainy & Rainy & & Cloudy & & \\
\hline Time of Liquid Chlorine addition & 7:00 PM & 7:00 PM & 7:00 PM & 7:00 PM & 7:00 PM & 7:00 PM & 7:00 PM \\
\hline Quantity Added Kg & 5 & 5 & 4 & 4 & 3 & 3 & 2 \\
\hline Theoretical Cl2 ppm & 12.9 & 12.9 & 10.3 & 10.3 & 7.7 & 7.7 & 5.1 \\
\hline \multicolumn{2}{|c|}{ Measured C12@8 AM Next day } & 3.0 & 2.8 & 2.8 & 2.6 & 2.6 & 2.6 \\
\hline Measured C2 @12 Noon & & 2.0 & 2.2 & 2.2 & 2.2 & 2.2 & 2.2 \\
\hline Measured Cl2@2PM & & 1.2 & 1.4 & 1.4 & 1.6 & 1.8 & 1.8 \\
\hline Measured C2 @4PM & & 0.6 & 1.0 & 1.2 & 1.0 & 1.2 & 1.4 \\
\hline Measured C2 @6PM & & 0.4 & 0.4 & 1.0 & 0.8 & 1.0 & 1.2 \\
\hline
\end{tabular}


Table 6. Monitoring of FAC in Club Pool : CG 14 ( Liquid Chlorine Operated)

\begin{tabular}{|c|c|c|c|c|c|c|c|}
\hline Pool Capacity KL & 600 & & & & & & \\
\hline Chlorinat ing Agent & \multicolumn{2}{|c|}{ Liquid Chlorine } & & & & & \\
\hline Run No & $\mathrm{R} \quad \# 029$ & $\mathrm{R} \quad \# 030$ & $\mathrm{R} \quad \# 031$ & $\mathrm{R} \quad \# 032$ & $\mathrm{R} \quad \# 033$ & $\mathrm{R} \quad \# 034$ & $\mathrm{R} \quad \# 035$ \\
\hline Run Date (Year 2010) & Sep-24 & Sep-25 & Sep-26 & Sep-27 & Sep-28 & Sep-29 & Sep-30 \\
\hline Maximum Temp Deg C & 43.0 & 43.0 & 44.3 & 43.1 & 44.1 & 45.6 & 47.1 \\
\hline Minimum Temp Deg C & 22.5 & 24.0 & 24.0 & 24.9 & 24.5 & 25.6 & 27.6 \\
\hline Relative Humidity $\%$ Evening & 65 & 100 & 63 & 60 & 67 & 55 & 57 \\
\hline Wind Velocity $\mathrm{Km} / \mathrm{Hr}$ Evening & 8.0 & 14.0 & 4.0 & 4.0 & 8.0 & 6.0 & 6.0 \\
\hline Rains if any / Remarks & & Rainy & Rainy & & Cloudy & & \\
\hline Time of Liquid Chlorine addition & 7:00 PM & 7:00 PM & 7:00 PM & 7:00 PM & $7: 00 \mathrm{PM}$ & 7:00 PM & 7:00 PM \\
\hline Quant ity Added Kg & 140 & 140 & 105 & 105 & 70 & 70 & 70 \\
\hline Theoretical Cl2 ppm & 29.2 & 29.2 & 21.9 & 21.9 & 14.6 & 14.6 & 14.6 \\
\hline \multicolumn{2}{|c|}{ Measured Cl2@8 AM Next day } & 3.0 & 2.8 & 2.6 & 2.8 & 2.8 & 2.6 \\
\hline Measured C2 @ 12 Noon & & 1.8 & 2.0 & 2.0 & 2.0 & 2.0 & 2.0 \\
\hline Measured C2 @2PM & & 1.2 & 1.4 & 1.6 & 1.6 & 1.4 & 1.6 \\
\hline Measured Cl2@4PM & & 0.6 & 0.8 & 1.0 & 1.2 & 1.2 & 1.4 \\
\hline Measured Cl2@6PM & & 0.2 & 0.4 & 0.6 & 0.8 & 1.0 & 1.0 \\
\hline
\end{tabular}

\section{Stabilised Condition in the Pool}

As could be seen from the above tables, the Liquid Chlorine operated pooltakes 6 to 7 days to stabilize, where as the TCCA operated pool is stabilised on day 4 for the requirement of minimum $1 \mathrm{ppm}$ in pool water. The observations are graphically represented in Figures \# 1 to Figure \# 5

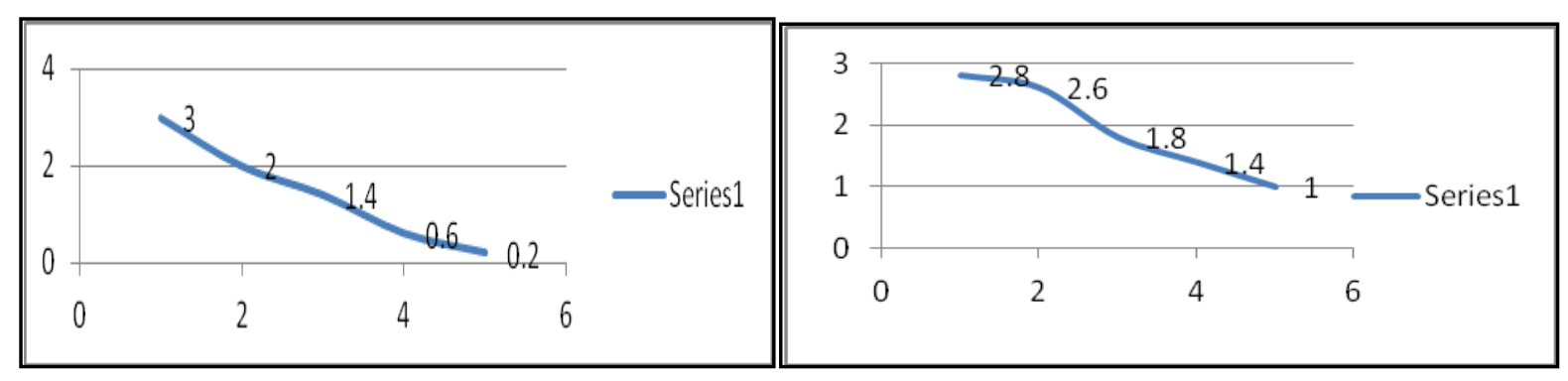

FAC Values Measured on Day \# 2

FAC Values Measured on Day \# 7 ( Stabilized)

Figure 1. Measured FAC values in Pool: AB 05 ( Liquid Chlorine Operated )
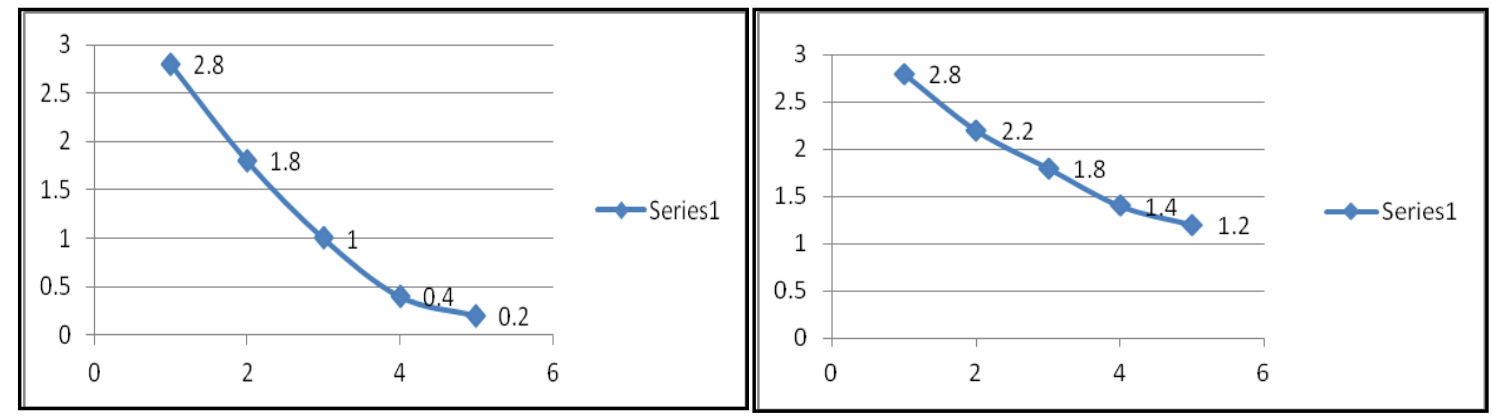

FAC Values Measured on Day \# 2

FAC Values Measured on Day \# 6 ( Stabilized)

Figure 2. Measured FAC values in Pool: PK 06 ( Liquid Chlorine Operated)
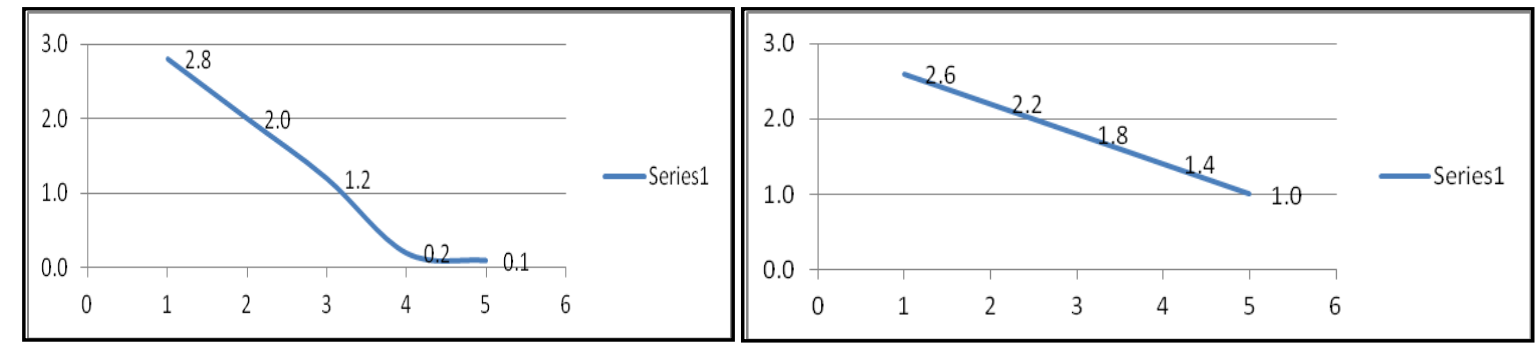

FAC Values Measured on Day \# 2

FAC Values Measured on Day \# 7 ( Stabilized )

Figure 3. Measured FAC values in Pool:PK 07 ( Liquid Chlorine Operat ed ) 


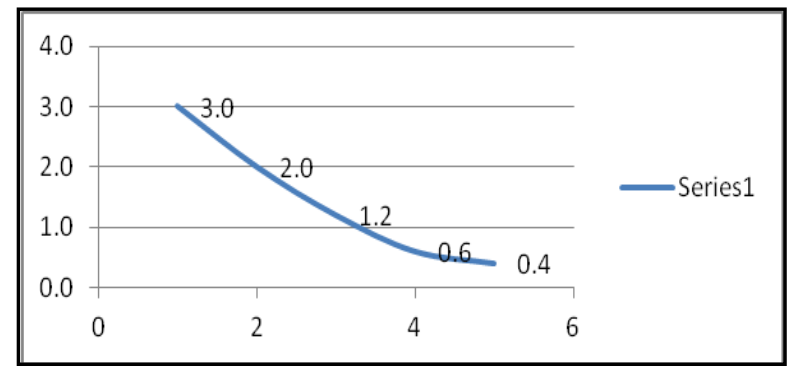

FAC Values Measured on Day \# 2

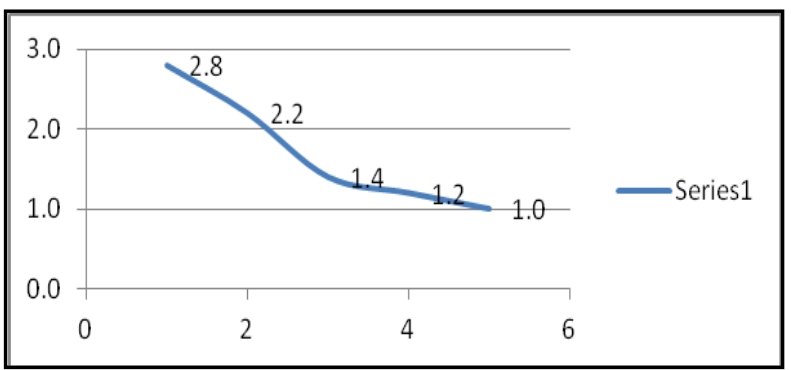

FAC Values Measured on Day \# 4 ( Stabilized )

Figure 4. Measured FAC values in Pool:PC 13 ( TCCA Operated )

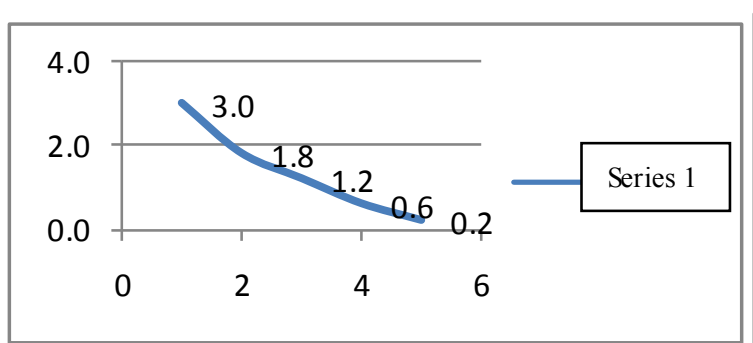

FAC Values Measured on Day \# 2

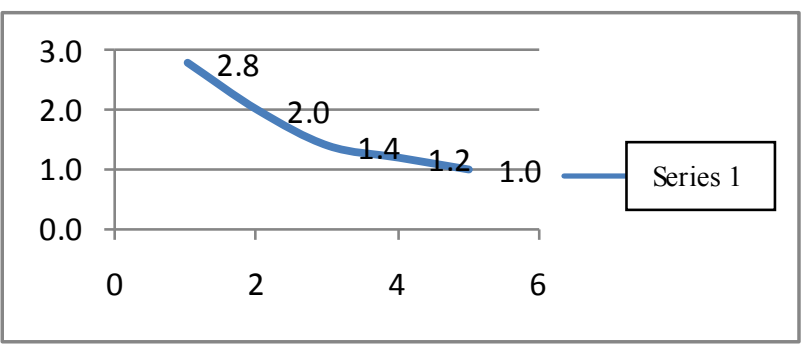

FAC Values Measured on Day \# 6 ( Stabilized )

Figure 5. Measured FAC values in Pool: CG 14 ( Liquid Chlorine Operated )

\section{Break Point Chlorination}

Prior to operation start ups, the pools were treated with the sanitization agent, the actual ppm of chlorine added to FAC was monitored to establish the Break point chlorination as represented in Figures \# 6 to \# 9

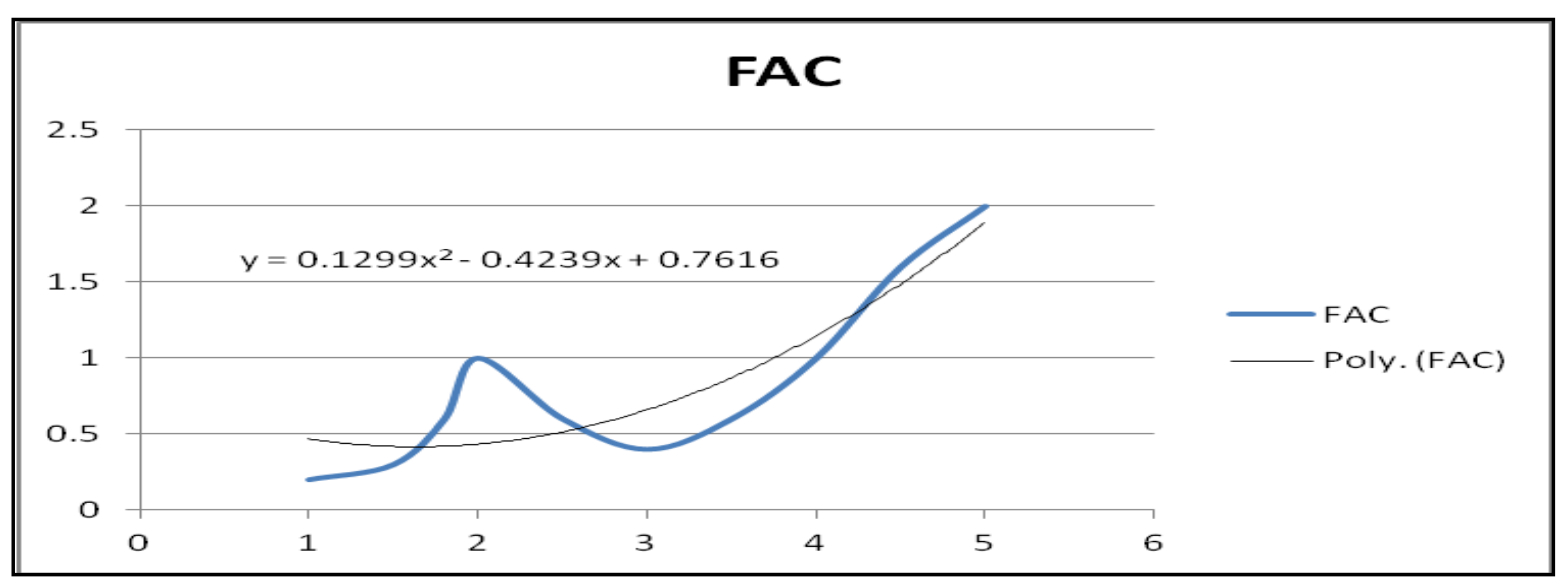

Figure 6. Break Point Chlorination - AB 05:300 KL Liquid Chlorine

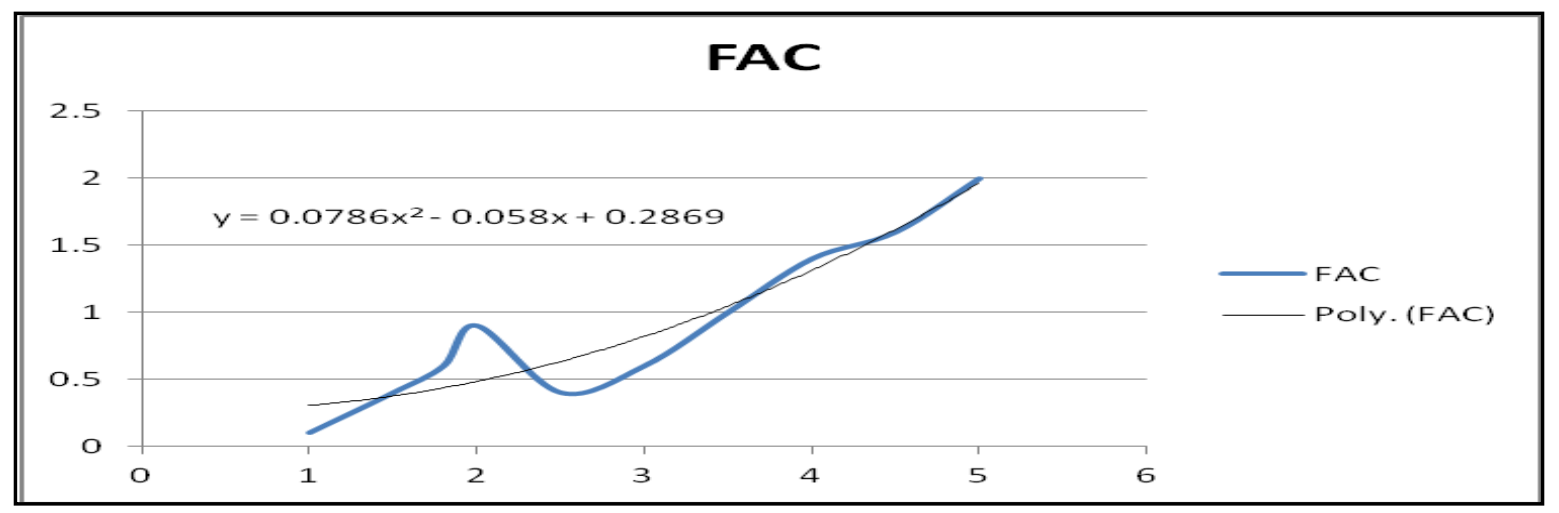

Figure 7. Break Point Chlorination - PK 07: 500 KL Liquid Chlorine 


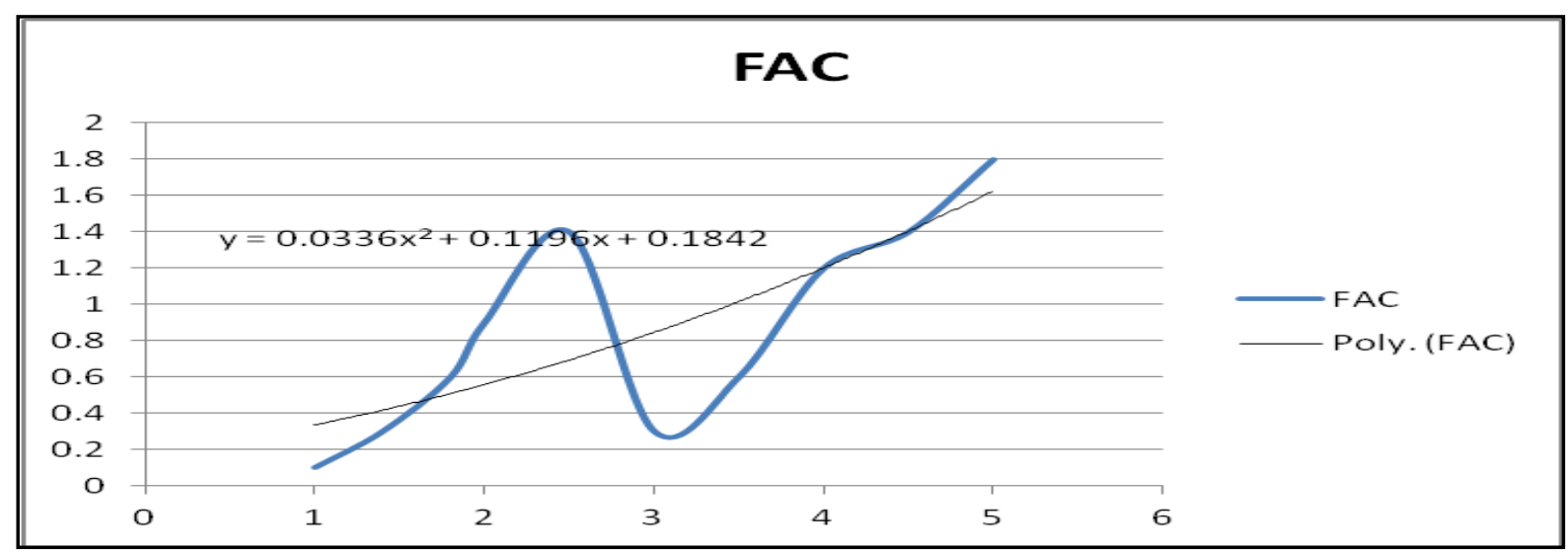

Figure 8. Break Point Chlorination - PC 13:350 KL TCCA

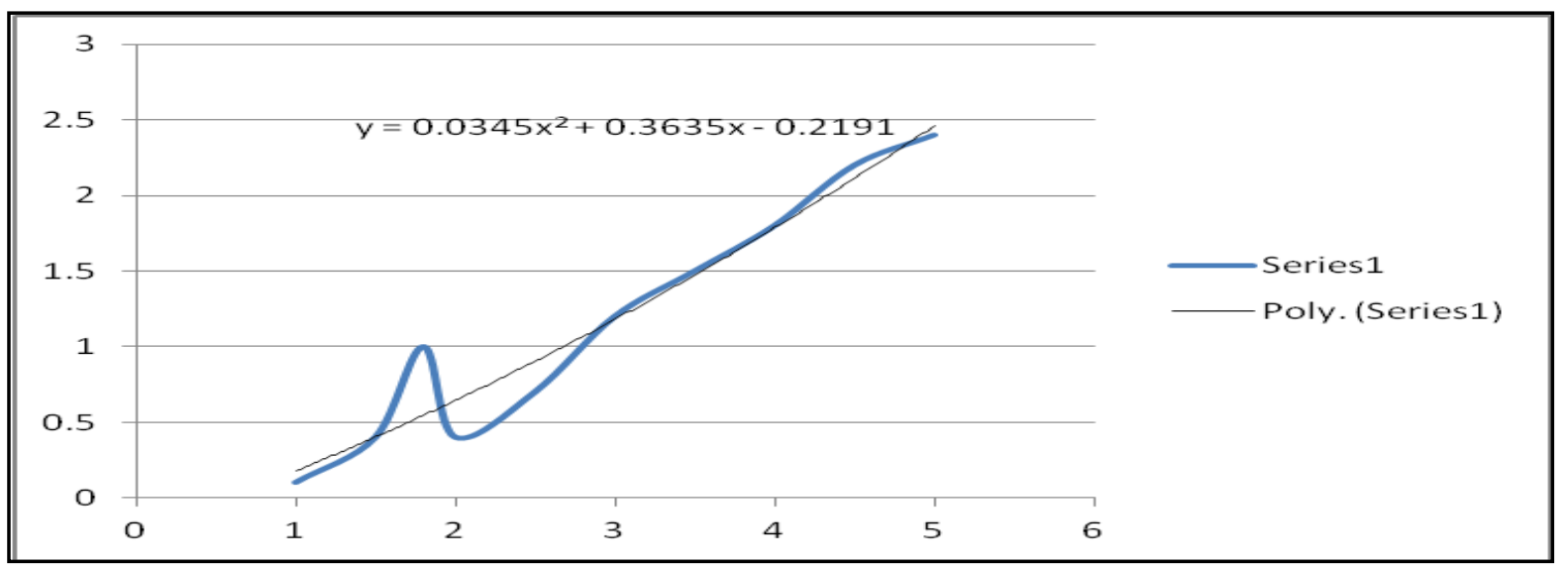

Figure 9. Break Point Chlorination - CG 14: 600 KL Liquid Chlorine

\section{Results and Discussions}

It was observed that the pool with TCCA got stabilized in day 4 itself, and hence all the Liquid Chlorine operated pools were treated with TCCA and as could be seen from Tables 7 to 10 , the pools got stabilized much earlier. TCCA Ch lorinating agent is added in the evening at 7 PM and FAC readings are taken the subsequent day at 8 AM, 12 Noon, 2 PM, 4 PM and 6 PM. The observations for Pools are reproduced in Table \# 7 to Table \# 10

Table 7. FAC Monitoring in Stabilised Pools ( AB 05) using TCCA

\begin{tabular}{|c|c|c|c|c|c|}
\hline Run No & $\mathrm{R} \# 036$ & $\mathrm{R} \quad \# 037$ & $\mathrm{R} \quad \# 038$ & $\mathrm{R} \# 039$ & $\mathrm{R} \quad \# 040$ \\
\hline Run Date (Year 2010) & Oct-01 & Oct -02 & Oct -03 & Oct-04 & Oct-05 \\
\hline Maximum Temp Deg C & 32.5 & 32.7 & 33.3 & 33.6 & 32.5 \\
\hline Minimum Temp Deg C & 20.3 & 22.0 & 22.8 & 23.0 & 22.8 \\
\hline Relative Humidity \% Evening & 64 & 61 & 75 & 91 & 58 \\
\hline Wind Velocity $\mathrm{Km} / \mathrm{Hr}$ Evening & 0.0 & 0.0 & 4.0 & 7.0 & 6.0 \\
\hline Rains if any / Remarks & & & & Traces & Rainy \\
\hline Time of TCCA 90 addition & 7:00 PM & 7:00 PM & 7:00 PM & 7:00 PM & 7:00 PM \\
\hline Quant ity of TCCA Adde d Kg & 3 & 2 & 1.5 & 1.5 & 1 \\
\hline Theoretical Cl2 ppm & 9.0 & 6.0 & 4.5 & 4.5 & 3.0 \\
\hline Measured C2 @8 AM Next day & & 2.8 & 2.4 & 2.4 & 2.2 \\
\hline Measured Cl2@12 Noon & & 2.2 & 2.2 & 1.8 & 1.6 \\
\hline Measured Cl2@2PM & & 1.6 & 1.8 & 1.6 & 1.4 \\
\hline Measured Cl2@4PM & & 1.2 & 1.2 & 0.8 & 1.0 \\
\hline Measured Cl2@6PM & & 0.8 & 0.8 & 0.6 & 0.8 \\
\hline
\end{tabular}


Table 8. FAC Monitoring in Stabilised Pools ( PK 06) using TCCA

\begin{tabular}{|c|c|c|c|c|c|}
\hline Run No & $\mathrm{R} \quad \# 041$ & $\mathrm{R} \quad \# 042$ & $\mathrm{R} \quad \# 043$ & $\mathrm{R} \quad \# 044$ & $\mathrm{R} \# 045$ \\
\hline Run Date (Year 2010) & Oct-01 & Oct-02 & Oct -03 & Oct-04 & Oct -05 \\
\hline Maximum Temp Deg C & 32.5 & 32.7 & 33.3 & 33.6 & 32.5 \\
\hline Minimum Temp Deg C & 20.3 & 22.0 & 22.8 & 23.0 & 22.8 \\
\hline Relative Humidity \% Evening & 64 & 61 & 75 & 91 & 58 \\
\hline Wind Velocity $\mathrm{Km} / \mathrm{Hr}$ Evening & 0.0 & 0.0 & 4.0 & 7.0 & 6.0 \\
\hline Rains if any / Remarks & & & & Traces & Rainy \\
\hline Time of TCCA 90 addition & 7:00 PM & 7:00 PM & 7:00 PM & 7:00 PM & 7:00 PM \\
\hline Quantity of TCCA Added Kg & 4 & 3 & 3 & 2 & 1.5 \\
\hline Theoretical Cl2 ppm & 9.0 & 6.8 & 6.8 & 4.5 & 3.4 \\
\hline Measured Cl2@8 AM Next day & & 3.2 & 2.8 & 2.8 & 2.4 \\
\hline Measured C12@12 Noon & & 2.4 & 2.2 & 2.4 & 2.0 \\
\hline Measured Cl2@2PM & & 1.6 & 1.8 & 1.6 & 1.6 \\
\hline Measured Cl2@4PM & & 1.2 & 1.4 & 1.2 & 1.2 \\
\hline Measured Cl2@6PM & & 0.8 & 1.0 & 1.0 & 0.8 \\
\hline
\end{tabular}

Table 9. FAC Monit oring in Stabilised Pools ( PK 07) using TCCA

\begin{tabular}{|c|c|c|c|c|c|}
\hline Run No & $\mathrm{R} \# 046$ & $\mathrm{R} \quad \# 047$ & $\mathrm{R} \quad \# 048$ & $\mathrm{R} \quad \# 049$ & $\mathrm{R} \# 050$ \\
\hline Run Date (Year 2010) & Oct -01 & Oct-02 & Oct-03 & Oct -04 & Oct -05 \\
\hline Maximum Temp Deg C & 32.5 & 32.7 & 33.3 & 33.6 & 32.5 \\
\hline Minimum T emp Deg C & 20.3 & 22.0 & 22.8 & 23.0 & 22.8 \\
\hline Relative Humidity $\%$ Evening & 64 & 61 & 75 & 91 & 58 \\
\hline Wind Velocity $\mathrm{Km} / \mathrm{Hr}$ Evening & 0.0 & 0.0 & 4.0 & 7.0 & 6.0 \\
\hline Rains if any / Remarks & & & & Traces & Rainy \\
\hline Time of TCCA 90 addition & 7:00 PM & 7:00 PM & 7:00 PM & 7:00 PM & 7:00 PM \\
\hline Quant ity of TCCA Added Kg & 5 & 4 & 3 & 2 & 2 \\
\hline Theoretical Cl2 ppm & 9.0 & 7.2 & 5.4 & 3.6 & 3.6 \\
\hline Measured Cl2@8AM Next day & & 2.8 & 2.4 & 2.6 & 2.6 \\
\hline Measured Cl2@12 Noon & & 2.4 & 2.0 & 2.2 & 1.0 \\
\hline Measured Cl2@,2PM & & 2.0 & 1.6 & 1.6 & 1.4 \\
\hline Measured Cl2@4PM & & 1.2 & 1.0 & 1.2 & 1.2 \\
\hline Measured Cl2@6PM & & 0.8 & 0.8 & 1.0 & 1.0 \\
\hline
\end{tabular}

Table 10. FAC Monitoring in Stabilised Pools ( CG 14) using TCCA

\begin{tabular}{|c|c|c|c|c|c|}
\hline Run No & $\mathrm{R} \# 051$ & $\mathrm{R} \quad \# 052$ & $\mathrm{R} \quad \# 053$ & $\mathrm{R} \quad \# 054$ & $\mathrm{R} \quad \# 055$ \\
\hline Run Date (Year 2010) & Oct-01 & Oct-02 & Oct-03 & Oct-04 & Oct -05 \\
\hline Maximum Temp Deg C & 32.5 & 32.7 & 33.3 & 33.6 & 32.5 \\
\hline Minimum T emp Deg C & 20.3 & 22.0 & 22.8 & 23.0 & 22.8 \\
\hline Relative Humidity \% Evening & 64 & 61 & 75 & 91 & 58 \\
\hline Wind Velocity $\mathrm{Km} / \mathrm{Hr}$ Evening & 0.0 & 0.0 & 4.0 & 7.0 & 6.0 \\
\hline Rains if any / Remarks & & & & Traces & Rainy \\
\hline Time of TCCA 90 addition & 7:00 PM & 7:00 PM & 7:00 PM & 7:00 PM & $7: 00 \mathrm{PM}$ \\
\hline Quantity of TCCA Added Kg & 6 & 5 & 4 & 3 & 3 \\
\hline Theoretical Cl2 ppm & 9.0 & 7.5 & 6.0 & 4.5 & 4.5 \\
\hline Measured C2 @8 AM Next day & & 3.0 & 2.6 & 2.4 & 2.6 \\
\hline Measured Cl2@12 Noon & & 2.6 & 2.2 & 2.0 & 2.2 \\
\hline Measured Cl2@2PM & & 2.2 & 1.8 & 1.6 & 1.6 \\
\hline Measured Cl2@4PM & & 1.4 & 1.2 & 1.2 & 1.2 \\
\hline Measured Cl2@6PM & & 1.0 & 0.8 & 1.0 & 1.0 \\
\hline
\end{tabular}

\section{Summary}

The following observations are concluded from the experiments
1. Liquid Chlorine operated pool AB 05 of $300 \mathrm{KL}$ capacity was stabilised with 35 Litres on the 7 th day from start up. With a theoretical Chlorine level of about $30 \mathrm{ppm}$, the observed values in early next day was around $2.8 \mathrm{ppm}-$ 
resulting in heavy losses of Chlorine to the atmosphere. This is one of the reasons for local neighbours complain about.

2. Similarly Liquid Chlorine operated pool PK 06 of 400 KL capacity was stabilised with 35 Litres on the 6th day from start up. With a theoretical Chlorine level of about 30 ppm, the observed values in early next day was around 2.8 ppm - resulting in heavy losses of Chlorine to the atmosphere

3. Liquid Chlorine operated pool PK 07 of $500 \mathrm{KL}$ capacity was stabilised with 70 Litres on the 7 th day from start up. With a theoretical Chlorine level of about $30 \mathrm{ppm}$, the observed values in early next day was around $2.8 \mathrm{ppm}-$ resulting in heavy losses of Chlorine to the atmosphere.

4. Liquid Chlorine operated pool CG 14 of $600 \mathrm{KL}$ capacity was stabilised with 70 Litres on the 6th day from start up. With a theoretical Chlorine level of about $30 \mathrm{ppm}$, the observed values in early next day was around $2.8 \mathrm{ppm}-$ resulting in heavy losses of Chlorine to the atmosphere .

5. Whereas the TCCA operated pool PC 13 of $350 \mathrm{KL}$ capacity was stabilised with $3 \mathrm{Kg}$ TCCA on the $4^{\text {th }}$ day from start up. With a theoretical Chlorine level of $12 \mathrm{ppm}$, the observed values in early next day was around $3 \mathrm{ppm}$

6. During the break point analys is it was seen that with
Liquid chlorine for 3 to $4 \mathrm{ppm}$ added chlorine, the available was 1 ppm FAC, with TCCA it was less than 2 ppm added chlorine to get 1 ppm FAC. Moreover with Liquid the pool struggles to stabilise where as with TCCA it is much easier

7. After converting the Liquid operated pool to TCCA, the pool management had savings potential up to Rs 4,000per month per ONE Lakh litre capacity pool

\section{REFERENCES}

[1] Matter Doug De La : Swimming Pool Chemistry, classroom notes of Canada University, ( 2000 )

[2] Canelli Edmondo: Chemical, bacteriological ... properties of Cyanuric acid as applied to Swimming Pool disinfection: APHJ, USA Vol 64, No 2 , February (1974)

[3] Naraporn H, Nisakorn T Et5 al, Effect of TCCA Disinfectant in swimming pool water; The Joint International conference on Sustainable energy and environment December $1-3$, Thailand ( 2004 )

[4] NEERI Laboratory Manual 